\title{
Assessment of Cognitive Radio Networks through Military Capability Development Viewpoint
}

\author{
Topi Tuukkanen \\ Information Technology Division \\ Finnish Defence Research Agency \\ Riihimäki, Finland \\ topi.tuukkanen@mil.fi
}

Timo Bräysy

University of Oulu

Oulu, Finland

\author{
Stefan Couturier \\ Fraunhofer FKIE \\ Wachtberg, Germany \\ Jaroslaw Krygier \\ Military University of Technology \\ Warsaw, Poland \\ Vincent Le Nir \\ Royal Military Academy \\ Brussels, Belgium
}

\author{
Boyd Buchin \\ Rohde \& Schwarz \\ Munich, Germany \\ Erik Verheul \\ Royal Netherlands Navy \\ Ministry of Defence \\ Den Helder, Netherlands \\ Niels Smit \\ Ministry of Defence \\ Utrecht, Netherlands
}

\begin{abstract}
In the absence of real-life implementations, this paper attempts to conceptually capture potential benefits and improvements that Cognitive Radio Networks (CRN) could introduce to a military environment. Functional business capability areas, as well as a system model, are used to frame analysis. Military CRNs improve reliability and availability of information flows. These may lead to improved information sharing and situation awareness and eventually to wider adoption of mission command and self-synchronization. However, fundamental capacity benefits of the CRNs remain a research topic. The requirement of computer-aided planning, preparation and simulation environment in support of deployed CRNs could also serve as a research and development platform for a Cognitive Radio System development activity as a collaborative international effort.
\end{abstract}

Keywords- cognitive radio; cognitive radio system; cognitive radio network; cognitive radio ad hoc network; military capability, capability area, capability lines of development, DOTMLPFI

\section{INTRODUCTION}

Reference [1] posits that future military network requirements include being operated in an untrustworthy world, supporting more demanding applications, adapting to service provision differentiation (e.g., Federated Mission Networks [2]) as well as including more automation and near-zero configuration to lessen the burden on end-users.

Cognitive Radio (CR) has been proposed as a paradigm to resolve the dilemma between the ever-increasing demand for bandwidth and perceived spectrum scarcity. Although the term Cognitive Radio is well established, the notion of Cognitive Radio Networks (CRN) is much more volatile as will be presented later in this section.

Cognition, in general, is the ability to process knowledge, which comprises tasks like memorizing, decision making, and learning. When applied to CR, this knowledge is mainly related to aspects of radio environment, user requirements, or policies. Drawing from the advances in Software Defined Radio technology, the notion of CR was first introduced by [3] and has been further elaborated, e.g., in [4].

This paper is based on NATO IST Panel activity IST-140 RTG-065 on Cognitive Radio Networks - Efficient Solutions for Routing, Topology Control, Data Transport, and Network Management.
Since its inception, the notion of CR has evolved within various standardization processes. In this article, the term CR shall be used as a "node", as a singular element within the system, instead of a "radio unit" or "radio device". Based on these, the concept is further expanded in [5] by the definition of the term Cognitive Radio System (CRS) as:

"A radio system employing technology that allows the system to obtain knowledge of its operational and geographical environment, established policies and its internal state; to dynamically and autonomously adjust its operational parameters and protocols according to its obtained knowledge in order to achieve predefined objectives; and to learn from the results obtained."

According to [6] the general concept of cognitive networking was first introduced in [7] under the concept of knowledge plane including all the major components of the later cognitive networking paradigm. However, the term CRN has multiple meanings in the literature.

A significant amount of research is based on the view that CRNs inherit the CRs' objectives of improving spectrum efficiency by a Secondary User (SU) accessing underutilized parts of the spectrum of the licensee or the Primary User (PU). This would mean that a CRN is a network of CRs operated by $\mathrm{SU}$ in the spectrum of the licensed PU. In such cases, the SU would be mandated to avoid interference to $\mathrm{PU}$ or to abandon that specific part of spectrum altogether [8]-[10].

In [11] the authors present a scheme where CRs shall form a network of cognitive radios. Reference [12] observes how the notion of $\mathrm{CR}$ has evolved and provides a generalized definition of Cognitive Radio Network essentially drawing from the spectrum-oriented definition of the CR. On the other hand, [13] expands the notion of cognition to include network layer functionalities and defines $\mathrm{CRN}$ as:

"A type of radio network in which the behavior of each radio is controlled by cognitive control mechanism to adapt to changes in topology, operating conditions, or user needs."

Reference [14] posits that a cognitive radio network is a multi-hop wireless heterogeneous network, allowing peer-topeer communications. That may involve the use of different 
types of radios, and [14] further argues that when CRs are connected to form a CRN, they may share local observations to support distributed decision making, thus cognition becoming a function of the network, rather than an individual radio. Although recognizing the above mentioned, [11] further posits that a CRN can be formed by a mix of cognitive and non-cognitive radios, if the cognitive function can instruct and control non-cognitive nodes.

Reference [15] further makes a distinction between node level and network level cognitive processing especially regarding the cognitive radio ad hoc networks. As [16] posits, the notion of CRN can be extended to unlicensed bands and also to ad hoc networks. As early as 2006, [17] has already observed that Cognitive Radio Networks can be either infrastructure based or infrastructure-less ad hoc networks and can expand over a number of licensed as well as unlicensed spectrum bands.

In this article our specific focus is on the military application of CRNs, bearing in mind that an obvious approach would be to do so as a Cognitive Radio Ad Hoc Network (CRAHN). We recognize that many aspects to be considered may also be elements of Contemporary Combat Net Radio Systems or Mobile Ad-hoc Networks (MANET). Major differences between traditional ad hoc networks and CRAHNs can be categorized in terms of choice of transmission spectrum, topology control, multi-hop/multispectrum transmission, and distinguishing node mobility from channel handoff enforced by PU activity [18].

To summarize, in this article we shall adhere to the broad and generic definition of CRN by the IEEE standard above, recognizing the difference between the optimization of objectives at local node and network levels.

As [19] suggests, there seems to be an open research gap between the military art of war and modern radio engineering disciplines. Military expectations, needs, and formal requirements have not been elaborated in the publicly available academic documentation, whereas engineering efforts and potential new capabilities have not been crossreferenced nor analyzed to meet those military requirements. Therefore, this article continues the effort to bridge these two communities of interest.

Our paper is structured as follows. In the first section, we introduced the notion of Cognitive Radio Networks. In the next section, we present characteristics of a military CRN together with the capability hierarchy and system model used for our analysis as the basis for our analytical approach. In the third section, a military CRN is subjected to an assessment through a military capability viewpoint. Our observations are summarized in the final section.

\section{ANALYTIC APPROACH APPLIED IN THIS STUDY}

\section{A. Characteristics of a military $C R N$}

CRs are an essential component of cognitive networks. At individual Cognitive Radio/Node level, the solution of local optimum by the local cognitive process may be mathematically tractable but is of limited use especially if conflicting with network level overall end-to-end objectives. Within cognitive networking, learning is applied vertically across the whole protocol stack, and horizontally across the nodes in a network topology. It is in this full cognitive networking that [20] considers cognition to provide networks with unconstrained adaptability, capacity, reliability, and scalability. In addition, expanding from [21] we infer that a military $\mathrm{CRN}$ is to optimize the performance of the network given the constraints of multiple protocol stack layers in potentially diverse radio environments.

Reference [22] identifies several yet unresolved issues in military CRNs that are being further elaborated in ongoing NATO Science and Technology Organization's IST-140 Research Task Group, to which this paper also aims to contribute. Within the same initiative, [23] notes that the number and types of metrics and configuration parameters are highly dependent on the actual protocols and waveforms implemented within the CRN. Reference [23] continues with a further analysis of relevant $\mathrm{CRN}$ technologies such as routing, topology control and clustering, data transport, network management, trust management, and exchange of control information (control channel).

Attempts to optimize performance under such challenging conditions will potentially lead to a crosslayered architecture. An organizing entity, usually referred to as the Cognitive Engine, is required to take the decisions based on the metrics of all elements and to set the configuration parameters according to the decisions [24].

For any new technology under development, the objective should be to meet a need in the best possible for the least cost. Thereby a CRN should provide, over an extended period, better end-to-end performance than a noncognitive wireless network [25]. Reference [26] notes that there are four levels at which metrics can be measured: terminal level (a range of single radio metrics), link level (a range of metrics to be measured between two terminals), network level (aggregate or statistical metrics that can be measured over several nodes and links), and cognitive level (overall system-wide aggregate behavior).

Individual cognitive radio nodes perform numerous measurements and assess their own communications situation at local level cognitive processes that consume policies, which are already stored in their local databases, but also produce large numbers of measurement data that also needs to be provided for the network level cognitive processes. It is reasonable to expect that a military CRN will be designed in such a manner as to use local measurements in a form not overloading network level processing and storage capabilities. Furthermore, it seems that military CRNs cannot be designed upon contemporary notions of big data or cloud storage, because of mobility requirements and potential battlefield damage to the fixed communications infrastructure. Therefore, the information and knowledge management aspect needs to be considered in parallel with the design of cognitive spectrum functions and CRN design.

Several key parameters of the CRN must be identified to accurately reconfigure, adapt, and optimize the nodes within the network. Similarly, military operational analysis of different alternative approaches to the development and design choices regarding CRNs would significantly benefit from the identification of Key Measures of Performance [27].

Reference [28] addresses power control at the physical layer, scheduling at the link layer, and flow routing at the network layer. They also present mathematical models to characterize the interdependency among power control, scheduling, and routing, facilitating inter alia throughput maximization in a multihop CR network as well as better understanding of cross-layer design issues.

The source burstiness and end-to-end delay are significant components in the study of cognitive radio networking, although they have little impact on traditional 
Shannon theory. To unleash the potential of cognitive radios, practical protocols that address both end-to-end delay and source burstiness and network robustness are yet to be designed [29]. Reference [30] concludes that applying cognition or increasing side information available to the cognitive transceivers increases the amount and improves the quality of the communication.

Environment measurements that are used to optimize the system, as well as configuration variables, e.g., transmission controls, are yet to be defined. Similarly, a real-time or nearreal-time control process for optimization and QoS provisioning needs to be specified. One approach to implement the reasoning, decision making and learning aspects within the cognitive process is to employ Artificial Intelligence (AI) methods. Variables that feed the AI process include, e.g., transmission power and modulation type, but values of these are also outputs of the cognitive engine to optimize the communication objectives of the system. Besides the environmental data used to model the channel and the transmission parameters, variables with potential value space must be defined to describe and measure the communication performance objectives. Such variables facilitate the Cognitive Engine to control the system to a specific state. The range and nature of such variables selected to be used within the Cognitive Engine may significantly influence the quality and efficiency of the engine [27].

At networking level, some of the goals can be described with policies or constraints, yet some of the objectives must be expressed in a form that is quantitative and can be used for mathematical optimization. One way to accomplish this is to attach a utility function to a function or process, thereby mapping the measurable attributes to a numerical value. Typical examples of key attributes of network connections include throughput, delay, cost, power consumption, and various error rates [6].

The requirement for military CRNs to support legacy networks and protocol stacks makes it necessary to build modular and open interface-based information exchange as an additional architectural component instead of being architecture itself [6]. Subsequently, they posit that the most significant design decision on the architectural level is the choice of the overall communication paradigm, arguing that a more data-centric approach by publish-subscribe type of the signaling would dramatically change information flows within a network.

Reference [6] suggests, supported by [31], a modular design including modules for topology control, routing, medium access (MAC) as well as decision making. These modules would thereby:

- learn from spectrum availability, link stability, path stability and traffic patterns;

- use available spectrum and channels;

- control topology and utilize spectrum efficiently;

- induce topology, routes, and paths of information flows;

- test the performance of potential configuration choices before implementing.

On the other hand, [32] lists dozens of studies of CRNs with different design goals, e.g., maximizing throughput or route stability, minimizing delay or route recovery/maintenance cost, most of which are based upon relatively static scenario or configuration. Many such studies will not yield militarily useful results, especially in case hostile enemy actions (kinetic + non-kinetic) need to be considered.

In our attempt to find general indicators of performance of CRNs that could be used to develop a military operational understanding of potential CRN design choices, [33] has presented five generic categories of wireless network resources that could be used to define and select appropriate metrics. These resource categories are:

- radio resources, including time, frequency, space, and power/energy;

- built-in resources such as mass storage, batteries, and processing units, e.g., CPUs;

- user interface resources, which refer to sensors as well as actuators integrated into wireless terminals like speakers, microphones, or imaging devices;

- social resources, which include the individuals behind the wireless devices (e.g., controlling or taking decisions), as well as groups of them;

- connectivity resources, which typically include several cellular and short-range links.

Reference [26] argues that regarding network metrics, scalability becomes essential. Such generic metrics include, but are not limited to, the capacity of the network, session outage probability, throughput (the amount of packet transmissions without rejections and with a finite delay), goodput (the amount of useful data that make it through the system), delay, the number of users, probability of error (introduced by multiple users), header vs. payload, network compatibility (interoperability, heterogeneous vs. homogeneous), number of nodes, speed of nodes, types of nodes, time to reach network stability, and time to reconfigure the network.

Besides the metrics or features already observed, [34] further posits that within wireless networking the objectives for optimization are often competing. It further addresses four specific metrics of capability, reliability, scalability, and density, concluding that such metrics are not altogether independent, nor orthogonal. In addressing these metrics, the reference further introduces various system features such as interference tolerance, matching range and usage, power management with dynamic usage, use of higher frequencies, and receiver performance.

On the other hand, within the discipline of System Engineering, general military performance categories have been presented by [35], involving those of performance (capability, behavior), availability (reliability, maintainability), adaptability (flexibility, expandability), interoperability (communication, protocol), usability (human factors, man-machine interface), survivability (avoidance of detection, self-defense, damage-tolerance), security (data, physical), and safety (development, operation, maintenance, disposal).

However, the very existence of an optimum balance between adaptation and performance has been challenged by, e.g. [36]. As mitigation to the previous argument, [37] has found that by a trade-off between robustness, performance, and complexity, a stable state can be achieved. Reference [33] presented five generic categories of wireless network resources that could be used to define and select appropriate metrics. Furthermore, [26] argues that regarding network metrics, scalability becomes important. Besides metrics or features already observed, [34] further posits that within wireless networking the objectives for optimization are often competing. It concludes that one of the 
fundamental research needs yet remaining is "what are the fundamental capacity benefits" to be gained, continuing that absolute measures of network capacity in a broad range of scenarios may not be available.

Therefore, in the absence of generally accepted key measures of performance, we shall provide a high-level description of a potential military CRN, implemented as a CRAHN, to frame our analysis. This shall be conducted using hypothetical capability statements:

- military CRNs are to provide mission, circumstances and environmentally balanced reliable and trustworthy communications capability that is self-organizing, delayand disruption tolerant, as well as self-healing.

- military CRNs (or sub-segments/clusters therein) can operate in several different operating modes simultaneously, being themselves the primary network exclusively using licensed (military assigned) frequencies (i.e. either implementing Dynamic Spectrum Access/Dynamic Spectrum Management paradigm or defining PUs and SUs internally within armed forces).

- military CRNs may operate as a secondary network using unused portions of spectrum licensed to other users (i.e., the traditional notion of Cognitive Radio Networks).

- military CRNs may operate as an inter-networking element between other primary and secondary networks.

- military CRNs may include cognitive radio nodes as well as non-cognitive radio nodes whose behavior the network may control.

- a military CRN can also include non-cognitive legacy radio nodes, waveforms, or links whose behavior is not controlled (e.g., legacy radio relay for trunking backbone)

- a military CRN may consist of handhelds, vehicular radios, as well as fixed radio nodes whose size, power consumption, heat dissipation, processing capabilities, complexity, antennae, available portions of the spectrum, and cost may vary.

- military CRNs can control individual nodes or clusters of nodes in such operating modes as to minimize the probability of interception or detection within specific military operational circumstances. Individual nodes or clusters of nodes can be operated in modes to circumvent, counter, dodge, or evade hostile electronic warfare measures.

- military CRNs' policies, constraints, and operating parameters can be planned and tested before implementation at an appropriate command level staff function, and they can be injected/inserted to nodes either locally or over-the-air as military circumstances dictate. The policies can be adjusted during the mission (as a result of a network operation or military conditions), and military $\mathrm{CRNs}$ should be ready to update them dynamically.

- a military CRN has to be aware that surrounding networks can also be equipped with cognitive features aiming at the same goal, for example within a multinational federated mission network. Military Cognitive Radio System must foresee such multi-CRNs operations to globally optimize the resources during the mission.

\section{B. Military Capability}

The concept of capability is used in different levels of planning and by different interest groups. To support these varied needs, several capability models have been introduced. Thereby some capability models represent certain perspectives of capability in a specific context and may lead to misunderstandings between stakeholders engaged in planning, building, maintaining or operating the military capability [38].

To embrace the benefits of various viewpoints and to avoid pitfalls of some capability models, a Comprehensive Capability Meta-Model (CCMM) was proposed in [39] and partially tested within the CR research and development community by [40]. In this paper, we shall continue these earlier works by assessing the notion of CRNs at CCMM's level 2 (Business Model) and level 3 (System Model).

In the capability-based planning, the capability is seen as an ability or a capacity to perform a set of tasks, or an ability to achieve the desired effect [41]. This functional, or business, perspective, and corresponding capability models are used to avoid potential bias to a particular capability solution and to develop solutions suitable for wide range of operations in different geographical locations [42]. To support the Capability Based Planning process, several predefined functional capability taxonomies are defined, such as US Joint Capability Areas (JCA) [41] and NATO Bi-Strategic Commands' Capability Hierarchy [43], the latter of which shall be used in our analysis as our business model.

Besides capability based planning, the acquisition community also uses the concept of capability, yet often viewing the concept of capability as systems. System models are typically used in the capability solution planning, building, and management. These models pay attention to all components of the capability instead of just focusing on the platforms and other technical components of capability.

Continuing our adaptation to the CCMM, we shall adhere to the level 3 system model consisting of components such as doctrine, organization, training, material, leadership, personnel, facilities and policy, as defined in the DOTMLPFI model [44]. Because of the heterogeneity of the components in this model, these system models are also referred as "lines of development" [38].

Within the CR research community, reference [45] collected and rearranged proposed technical properties and possible technical characteristics of cognitive radios. One of these property groups, dynamic spectrum access, was tested in [19] through DOTMPLFI. The role and place of such military assessment of technical characteristics within broader military capability based planning process were further elaborated in [40].

For our purposes, the business model and functional capability areas are best exemplified by [43]. Within NATO, the high-level military functional capability is divided into seven capability areas:

- $\quad$ Prepare (R)

- $\quad$ Project (D)

- Engage (E)

- $\quad$ Sustain (S)

- $\quad$ Protect $(\mathrm{P})$

- $\quad$ Inform (I)

- Consult, command and control (C).

These capability areas are further decomposed into one or more lower level capability tiers. 
The system model is known as DOTMLPFI. The use of the model has been described in more detail in [19], but to summarize, these lines of development are:

- a doctrine (D) is an expression of the principles by which military forces guide their actions and is a codification of how the activity is conducted.

- organization (O) relates to the operational and nonoperational organizational relationships of people.

- training $(\mathrm{T})$ is the provision of the means to practice, develop and validate, within constraints, the practical application of military doctrine to deliver a military capability.

- materiel (M) is the provision of military platforms, systems, and weapons, expendable and non-expendable, needed to outfit/equip an individual, group or organization.

- leadership (L) is influencing people by providing purpose, direction, and motivation while operating to accomplish the mission and improve the organization.

- personnel (P) relates the timely provision of sufficient, capable and motivated personnel to deliver defense outputs, both now and in the future.

- facilities (F) relates the acquisition, development, management, and disposal of all fixed, permanent buildings and structures, land, utilities, and facility management services in support of defense capabilities.

- interoperability (I) is the ability of armed forces and, when appropriate, forces of partner and other nations to train, exercise and operate effectively together in the execution of assigned missions and tasks.

Figure 1 depicts different disciplines of capability-based planning and highlights to the right the viewpoints of system and business models to be elaborated further in this article.

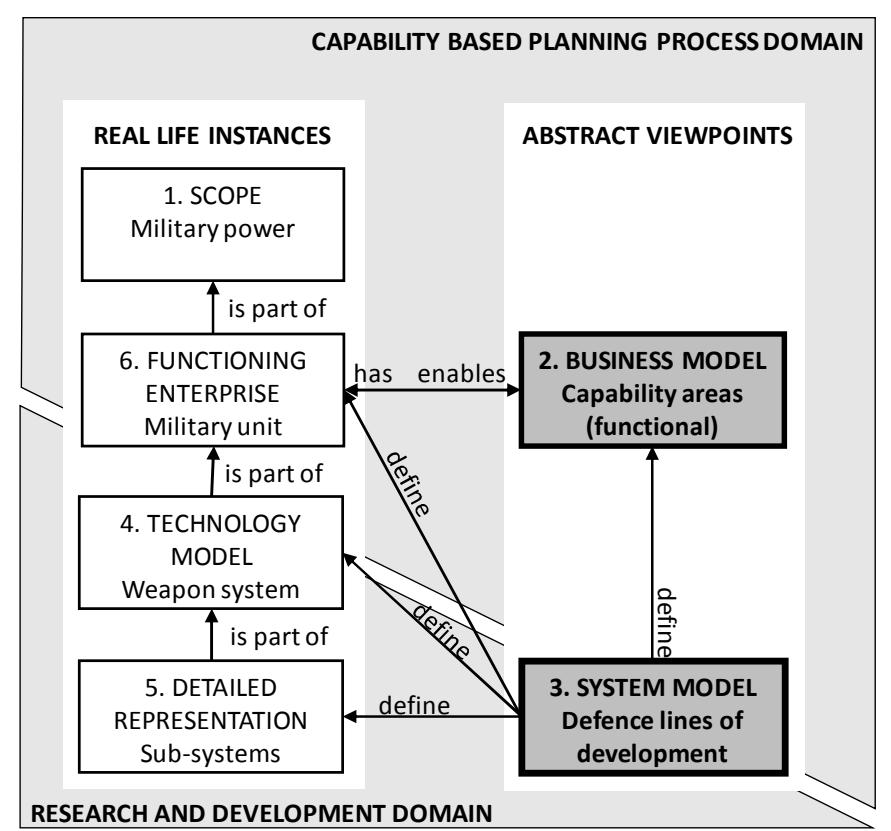

Figure 1. Capability perspectives and viewpoints and their relations to each other. Adopted from [40]. The figure depicts different viewpoints that can be adopted by different communities of interest within the military capability development process. Viewpoints applied in this article are highlighted to the right.

These two models above provide us the framework within which to conduct our assessment of the potential of CRNs to contribute to the military capabilities. As suggested in [46], we shall consider the life-cycle of a military CRN, if needed, compressed into three phases of pre-deployment (i.e. capability planning, procurement, and training), deployment (i.e. operations planning and implementation, monitoring, and maintenance), and post-deployment (i.e. withdrawal and cognition policies evaluation) phases.

Furthermore, we shall consider three organizational levels of the military apparatus involved in potential development, procurement and use of the military CRNs, namely those of high-command (e.g. MoD, Joint Staff), operations command (e.g. Operations Headquarters, Combined Joint Task Force), and tactical level (e.g. brigade, battalion, company, end users).

Before commencing our analysis of the potential impact that military CRNs could contribute to military capabilities, we shall note that although this analysis concentrates on the cognitive networking layer specifically, CRNs do also need cognitive spectrum functions of lower ISO/OSI-layers to achieve desired objectives. Based on the generic assessment of CRSs in [19] and [40], the cognition as a general feature is expected to:

- lessen planning burden and shorten planning time

- facilitate automated configuration changes leading to adaptability,

- improve the quality, availability, reliability, and timeliness of the information flows,

- adopt reliable, efficient and fast dynamic spectrum access schemes appropriate for the situation at hand,

- support improved interoperability, and

- allow intelligent implementation of both tactical offensive and defensive electronic warfare measures, if so required.

\section{CRN CONTRIBUTIONS TO MILITARY CAPABILITIES}

Within our business model, and notably within the capability area Prepare ( $R$ ), we observe that automation reduces training burden on operators and shifts emphasis to the preparations needed to promulgate policies that direct and guide cognition, thus contributing to lower tier of Force Preparation (R.1).

As cognition automatically implements node and network level technical "Lessons Learned" as well as local and network-wide "experimentation", and as it facilitates maintenance and implementation of national intra- and international technical "interoperability", we conclude that CRNs technically contribute to the tier of Capability Development (R.2) of the Prepare-capability area. The actual information exchange and communication services provided by a military CRN contribute directly to Corporate Management and Support -tier (R.7).

CRN's services and automated capability to adjust to the changing environmental factors support the capability area:

- Project and all its sub-tiers from mounting to deployment, staging and basing.

- Engage and notably the tiers of Joint Maneuver (E.1), Joint Fires (E.2). Pending on the practical implementation of different protocols and waveforms, the CRN may support Non-kinetic Engagement, for example by tactical self-protection jamming in conjunction with routing that supports the low probability of interception and detection. (E.3).

- Consult, Command and Control (C3) improving all aspects and tiers within.

- Protect pending on the practical implementation notably within sub-tiers of security (P.1), defense against hostile action (P.2), and hazard mitigation (P3.). 
- Inform by improving aspects and tiers of collection (I.1), processing (I.2), and dissemination (I.3).

Furthermore, within the components of our system model, we observe the following potential CRN induced implications to military capabilities in DOTMLPFI categories:

Doctrine: CRNs challenge armed forces in the predeployment phase especially by the elusive characteristics of cognition itself, which is difficult to quantify and specify for procurement by defense materiel administrations and acquisition offices. We recognize this as a potential future research topic. Within deployment phase, the perceived capability benefit from a CRN is improved availability and reliability of communications means. Besides natural and propagation challenges, CRNs may suffer from enemy kinetic and non-kinetic action, whereby, although reliable, the bandwidth requirements cannot always be met. Thus, a CRN is expected to inform users of bandwidth restraints. Doctrinally this improved reliability could, in time, lead to modifications in regular reporting mechanisms across multiple military hierarchy layers, i.e., moving from regular reporting to continuous on-line status monitoring, leading to improved information sharing and improved situation awareness. Such changes in standard operating procedures (SOP) can be foreseen, may be desirable, but are not mandatory nor forced by the adoption of CRNs. If CRNs employ artificial intelligence techniques such as reinforcement learning or genetic programming, deployment time, post-deployment data collection, analysis, and policy updates need to take place and should be reflected in doctrine and SOPs.

Organization: The detailed knowledge and understanding of particulars of networking functions within a CRN should be automated and cognitive, thus lessening the burden on individual end users. However, a CRN will need appropriately configured policies to function correctly. Thereby we foresee that functions of Signals Corps members shift at least one echelon higher than today to support the planning, preparation (pre-deployment), and surveillance (deployment) of the network instead of operating nodes themselves.

Training: Drawing again from the notion that CRNs in conjunction with cognitive spectrum management should make radio communications easier to use and more reliable for end users, we find it therefore reasonable to expect that training requirements of end users should decrease. Since there are a large number of end users, this should bring savings. The enhanced user experience together with improved reliability and increased availability may lead to secondary effects on behavior, reporting procedures, and military operational applications. However, since planning and preparations of policies become essential, the training load on those planners and network supervisors will increase moderately. As these planners and supervisors are expected to be members of Signals Corps, it is to be expected that such training can be conducted by modifications to their already existing curricula.

Materiel: As already postulated, a military CRN could consist of cognitive radio nodes either in end-user mode or as intermediate nodes within the network, implying a potential solution space that facilitates different device configurations from lightweight, power-constrained handhelds to a powerful multi-antenna vehicle-mounted versions. By the cognitive radio being an extension to the contemporary Software Defined Radio paradigm, most of the functionalities are expected to reside with the software, supported by databases containing measurement data as well as policies by which cognition is to be driven. A CRN can also be mostly software based. However, the network layer functionality would need network management functions that are expected to be operated at least one hierarchical layer above to the majority of end users. Conceptually a CRN may include non-cognitive radio nodes if the network control function may set configuration parameters to those nodes. This can be achieved by minor modifications to the already existing base of contemporary SDRs but may not apply to legacy combat net radios. Although CRNs are expected to perform well in various contingencies with their own policy data and measurements, updates to policies may need to be inserted into the system from time to time. Can major policy changes be conducted over the air? Can they be implemented and enforced during active military operations? These questions, among others, need to be properly addressed prior major acquisitions. Most importantly, the notion of cognition remains vague and under-specified within a military context, implying that military procurement agencies or materiel administrations are unable to place contracts or to start procurement for short- to mid-term future. This is a subject for future studies.

Leadership: As already alluded to, the CRNs, and more broadly CRSs, may have an impact on the regular reporting procedures. More importantly, the notion of reliable and available communications facilitates improved timely information sharing, which leads to improved situation awareness and may facilitate self-synchronization among military units. Self-synchronization, however, is dependent on commander's leadership style and the competencies of his subordinates and is, therefore, one possible outcome of the deployment of CRNs not precluding other leadership approaches should the commander choose otherwise [47]. Thereby we conclude that CRNs significantly contribute to the military $\mathrm{C} 2$ processes.

Personnel: The introduction of CRNs within the command and control structures may have a minor impact on the number of communications specialists and Signals Corps personnel needed especially in the lower end of the echelon as the ease of use allows regulars to operate Cognitive Radios with minimal training. However, predeployment planning and preparation of the policies CRNs need for proper functioning may need more thorough and indepth training, but as such functions are expected to be located higher in the echelon, numbers of such planners may be manageable, especially if combined with the role of network monitoring and supervision during the deployment.

Facilities: Memory and computational capacity needed to implement cognition are already available in high-end commercial handheld devices. Thereby, deployment of CRNs is not expected to give rise to any new space, weight, power, or heat requirements that advanced contemporary military radio systems would not already present. However, the network supervision and management, as well as planning and preparation of policies need software aided functionalities that may include some simulation capabilities to test policies before loading them to the network and devices. Such capabilities could precede the actual system development and procurement as the depicted planning, preparation, and simulation environment could also be used as a mechanism and a method for the overall CRN development activity itself. 
Interoperability: Communications interoperability was one of the initial drivers for the first software-defined radio applications in the military domain, and programs, such as ESSOR, are just about to prove the notion. Thereby we conclude that interoperability itself would not necessarily be the primary driver for the adoption of CRNs, although noting that in a CRN, individual nodes may be, for example, SDR based, and as such a CRN could facilitate and support interoperability.

\section{CONCLUSIONS}

In this paper, we explicitly concentrated on the potential future military application of the Cognitive Radio Networks. The research subject itself, a military CRN or a military Cognitive Radio System, does not yet exist, although a good number of limited scale demonstrations have already taken place. Thus the baseline of our study also incorporates the assumption that challenges inherent in contemporary Combat Net Radio Systems and MANETs are to be satisfactorily resolved in a military CRN.

In this article a notional military $\mathrm{CRN}$ was subjected to military capability development viewpoint, using capability areas as a business model and lines of development as a system model. We found that cognitive routing in military CRNs should choose a path minimizing interference to others, should be forward-looking in respect to linkavailability, and mitigate occurrences of rerouting to improve throughput and to decrease delay. Topology control is a combination of processes to construct and maintain topology, which is performed on link information provided by lower layers beneath ISO/OSI-networking layer. In military CRNs topology control mechanisms ensure good network energy efficiency, reduce energy consumption and rerouting, improve overall performance by minimizing delays, and maintain the connectivity of the CRN.

However, in our quest to find metrics or characteristic that could be used to assess the military potential of CRNs, a multitude of metrics and measures of performance were identified with sometimes conflicting observations regarding their applicability, e.g., regarding the metrics' scalability. Thereby we agree that fundamental capacity benefits of the CRNs remain a fundamental research topic. We observed that CRNs challenge defense materiel administrations and acquisition offices because the notion of cognition itself remains unclear and undefined.

One benefit we foresee by the implementation of military CRNs is improved reliability and availability that, through improved information sharing and improved situation awareness, may lead, but would not force so, to the adoption of mission command and military unit self-synchronization. Based on the generic description of CRN, cognition is expected to ease planning burden, to shorten pre-deployment time, and to facilitate automated configuration changes. These together:

- lead to adaptability,

- improve the quality, availability, reliability, and timeliness of the information flows,

- adopt reliable, efficient and fast dynamic spectrum access schemes appropriate for the situation at hand,

- support improved interoperability, and

- allow intelligent implementation of both tactical offensive and defensive electronic warfare measures, if required.

Large-scale adoption of CRNs is expected to shift functions of members of Signals Corps to higher echelon levels to support the planning, preparation (pre-deployment) and surveillance (deployment) of the network instead of operating devices and nodes themselves. CRNs are expected to perform well in various situations based on their own policies, data, and measurements. However, the question whether such policies could and should be adjusted or changed during operation should be addressed in future research. The requirement of computer-aided planning, preparation, and simulation environment was recognized, and development of such an environment could serve as a test and development platform for a broader combined CRS and CRN development activity.

This paper adopted a two-pronged approach to articulate potential benefits CRN technology could deliver, namely by the use of a business model (capability hierarchy) and a system model (lines of development). However, future CRNs may be based on a variety of design concepts, architectural approaches, and several protocol implementations, thus leading to a certain level of ambiguity in our conceptual analysis. This cannot be avoided until the international R\&D community starts to implement these different CRN building blocks for further scrutiny, e.g., through collaborative efforts suggested in the previous chapter.

\section{REFERENCES}

[1] R. Landry, K. Grace, and A. Saidi, "On the design and management of heterogeneous networks: a predictability-based perspective," IEEE Communications Magazine, vol. 42, no. 11, pp 80-87, 2004.

[2] NATO, "Federated Mission Networking Implementation Plan," 2014.

[3] J. Mitola and G. Q. Maguire, “Cognitive radio: making software radios more personal," IEEE personal communications, vol. 6, no. 4, pp. 13-18, 1999

[4] S. Haykin, "Cognitive radio: brain-empowered wireless communications," IEEE Journal on selected areas in communications, vol. 23, no. 2, pp. 201-220, 2005.

[5] Definitions of Software Defined Radio (SDR) and Cognitive Radio System (CRS) ITU-R SM.2152. ITU, 2009.

[6] P. Mähönen and J. Riihijärvi, "Cognitive radio network architectures," in Cognitive radio communications and networks: principles and practice, Wyglinski, A.; Nekovee, M.; Hou, T., Ed. Academic Press, 2009.

[7] D. Clark and et al., "A knowledge plane for the internet," in Proceedings of the 2003 conference on Applications, technologies, architectures, and protocols for computer communications, 2003, pp. $3-10$

[8] D. M. Alias and G. K. Ragesh, "Cognitive Radio networks: A survey," in 2016 International Conference on Wireless Communications, Signal Processing and Networking (WiSPNET), 2016, pp. 1981-1986.

[9] M. El Tanab and W. Hamouda, "Resource Allocation for Underlay Cognitive Radio Networks: A Survey," IEEE Communications Surveys\&Tutorials, 2016.

[10] A. Naeem and et al., "Network Coding in Cognitive Radio Networks: A Comprehensive Survey," IEEE Communications SurveysTutorials, 2017.

[11] B. A. Fette, Cognitive radio technology, $2^{\text {nd }}$ Ed., Academic Press, 2009.

[12] A. Wyglinski, M. Nekovee, and Y. T. Hou, Cognitive Radio Communications and Networks: Principles and Practice. Academic Press, 2009.

[13] Standard Definitions and Concepts for Dynamic Spectrum Access: Terminology Relating to Emerging Wireless Networks, System Functionality, and Spectrum Management, IEEE Std 1900.1. IEEE, 2008.

[14] H. Tang and S. Watson, Cognitive radio networks for tactical wireless communications. DRDC - Ottawa Research Centre, 2014

[15] L. Doyle and T. Forde, "The wisdom of crowds: cognitive ad hoc networks," in Cognitive Networks: Towards Self-Aware Networks, John Wiley\&Sons Ltd, 2007, pp. 203-221.

[16] M. Ibnkahla, Cooperative Cognitive Radio Networks: The Complete Spectrum Cycle. CRC Press, 2014. 
[17] I. Akyildiz and et al., "NeXt generation/dynamic spectrum access/cognitive radio wireless networks: A survey," Computer Networks, vol. 50, no. 13, pp. 2127-2159, 2006.

[18] I. F. Akyildiz, W.-Y. Lee, and K. R. Chowdhury, "CRAHNs: Cognitive radio ad hoc networks," AD hoc networks, vol. 7, no. 5, pp. 810-836, 2009.

[19] T. Tuukkanen and J. Anteroinen, "Framework to develop military operational understanding of cognitive radio," in International Conference on Military Communications and Information Systems (ICMCIS), Cracow, Poland, 2015, pp. 1-9.

[20] L. Kant and et al., "C-NEDAT: A cognitive network engineering design analytic toolset for MANETs," in IEEE Military Communications Conference (MILCOM), 2010, pp. 2333-2338.

[21] J. Sonnenberg and et al., "Quantifying the relative merits of genetic and swarm algorithms for network optimization in cognitive radio networks," in IEEE Military Communications Conference (MILCOM), 2012, pp. 1-8.

[22] S. Couturier and et al., "Challenges for network aspects of Cognitive Radio," in International Conference on Military Communications and Information Systems (ICMCIS), Cracow Poland, 2015, pp. 1-10.

[23] S. Couturier and et al., "End-to-End Optimization for Tactical Cognitive Radios," International Conference on Military Communications and Information Systems (ICMCIS), Bydgoszcz, Poland [submitted], 2018.

[24] R. Thomas and L. DaSilva, "Cognitive Networks," in Cognitive Radio Technology, 2nd Ed., Fette, B., Ed. Academic Press, 2009.

[25] H. Arslan, Cognitive radio, software defined radio, and adaptive wireless systems, vol. 10. Springer, 2007.

[26] NATO, "STO Technical report: Cognitive Radio in NATO, IST077," 2014.

[27] T. Newman, J. Evans, and A. Wyglinski, "Reconfiguration, adaptation, and optimization," Cognitive Radio Communications and Networks: Principles and Practice.(New York, NY:

Elsevier/North Holland), 2009.

[28] Y. Shi and Y. T. Hou, "Cross-layer optimization for multi-hop cognitive radio networks," in Cognitive radio communications and networks: principles and practice, Wyglinski, Alexander M and Nekovee, Maziar and Hou, Thomas, Ed. Academic Press, 2009.

[29] A. Goldsmith and et al., "Breaking spectrum gridlock with cognitive radios: An information theoretic perspective," Proceedings of the IEEE, vol. 97, no. 5, pp. 894-914, 2009.

[30] N. Devroye, "Information theoretical limits on cognitive radio networks," in Cognitive radio communications and networks: principles and practice, Wyglinski, Alexander M and Nekovee, Maziar and Hou, Thomas, Ed. Academic Press, 2009.

[31] O. Younis and et al., "Cognitive MANET design for missioncritical networks," IEEE Communications Magazine, vol. 47, no. 10, pp. 64-71, 2009

[32] L. Sun and et al., "Performance comparison of routing protocols for cognitive radio networks," IEEE Transactions on Mobile Computing, vol. 14, no. 6, pp. 1272-1286, 2015.

[33] M. Matinmikko and T. Bräysy, "Towards cognitive radio systems: main findings from the COGNAC project," VTT Research Notes, vol. 2575, p. 80, 2011.

[34] P. Marshall, "Scalability, Density, and Decision Making" in Cognitive Wireless Networks. Cambridge University Press, 2013.

[35] D. K. Hitchins, Putting systems to work, vol. 325. Wiley Chichester, 1992.

[36] K. M. Carley and Y. Ren, "Tradeoffs between performance and adaptability for C3I architectures," in 6th International Command and Control Research and Technology Symposium, 2001.

[37] N. Mokari and et al., "Robust ergodic uplink resource allocation in underlay OFDMA cognitive radio networks," IEEE Transactions on Mobile Computing, vol. 15, no. 2, pp. 419-431, 2016.

[38] J. Anteroinen, "Integration of existing military capability models into the comprehensive capability meta-model," in Systems Conference (SysCon), 2012 IEEE International, 2012, pp. 1-7.

[39] J. Anteroinen, "Enhancing the development of Military Capabilities by a Systems Approach,", Finnish National Defence University, 2013.

[40] J. Koivisto and T. Tuukkanen, "Comprehensive Capability Meta Model tested by a Cognitive Radio," in IEEE Military Communications Conference (MILCOM), Baltimore, MD, 2017, pp. 731-737.

[41] US DoD, "Directive: Capability Portfolio Management, Change $1, " 7045.20,2017$.

[42] “NATO Defence Planning Process," 2017. [Online]. Available: www.nato.int/cps/en/natolive/topics_49202.htm. [Accessed: 042017].
[43] NATO, "Bi-SC Capability Hierarchy," SH/PLANS/JCAP/FCP/15310118, 2015.

[44] ACT, "What is transformation? An Introduction to the Allied Command Transformation," 2015. [Online]. Available: www.ieee.es.

[45] T. Tuukkanen and J. Anteroinen, "Initial assessment of proposed cognitive radio features from a military perspective," 18 th International Command and Control Research and Technology Symposium, Alexandria, VA, 2013.

[46] T. Braysy and et al., "Network management issues in military cognitive radio networks," in International Conference on Military Communications and Information Systems (ICMCIS), Oulu, Finland, 2017.

[47] M. Vassiliou and D. Alberts, "C2 Failures: A Taxonomy and Analysis," in 18th International Command and Control Research and Technology Symposium, Alexandria, VA, 2013. 